Kurzbeiträge

Heinz Ahn

\title{
Controlling für Non-Profit-Organisationen - Quo vadis?
}

\author{
Anmerkungen am Beispiel des Krankenhaussektors
}

Balanced Scorecard; empirische Ergebnisse; Krankenhauscontrolling; Non-ProfitOrganisationen

Das klassische Controlling-Instrumentarium wird auch für den Einsatz in Non-ProfitOrganisationen herangezogen. Dabei werden die mit den Instrumenten verbundenen Prämissen allerdings oftmals nicht hinreichend hinterfragt. Der Beitrag zeigt die Problematik einer solchen Haltung auf und diskutiert dazu neue empirische Erkenntnisse zur Umsetzung der Balanced Scorecard im deutschen Krankenhaussektor. Die Ausführungen sollen - nicht zuletzt über eine kritischere Einstellung der Praxis - darauf hinwirken, Controlling-Instrumente stärker an die Besonderheiten des Non-Profit-Sektors anzupassen.

\section{Der Non-Profit-Sektor als Thema des Controllings}

Für Non-Profit-Organisationen (NPOs) werden die Ansätze und Konzepte der Betriebswirtschaftslehre von immer größerem Interesse. So vielfältig die einzelnen Gründe dafür auch sind, sie lassen sich im Wesentlichen auf die gleiche Ursache zurückführen: auf den verstärkten (markt)wirtschaftlichen Druck im Non-Profit-Sektor. Dies schlägt sich auch in Publikationen zum Thema nieder. So wurden zwölf verbreitete betriebswirtschaftliche Zeitschriften für den Zeitraum 1989-2004 auf Beiträge mit NPO-Bezug hin durchsucht. Insgesamt fanden sich immerhin 476 entsprechende Abhandlungen, deren zeitliche Verteilung in Abbildung 1 dargestellt ist. 


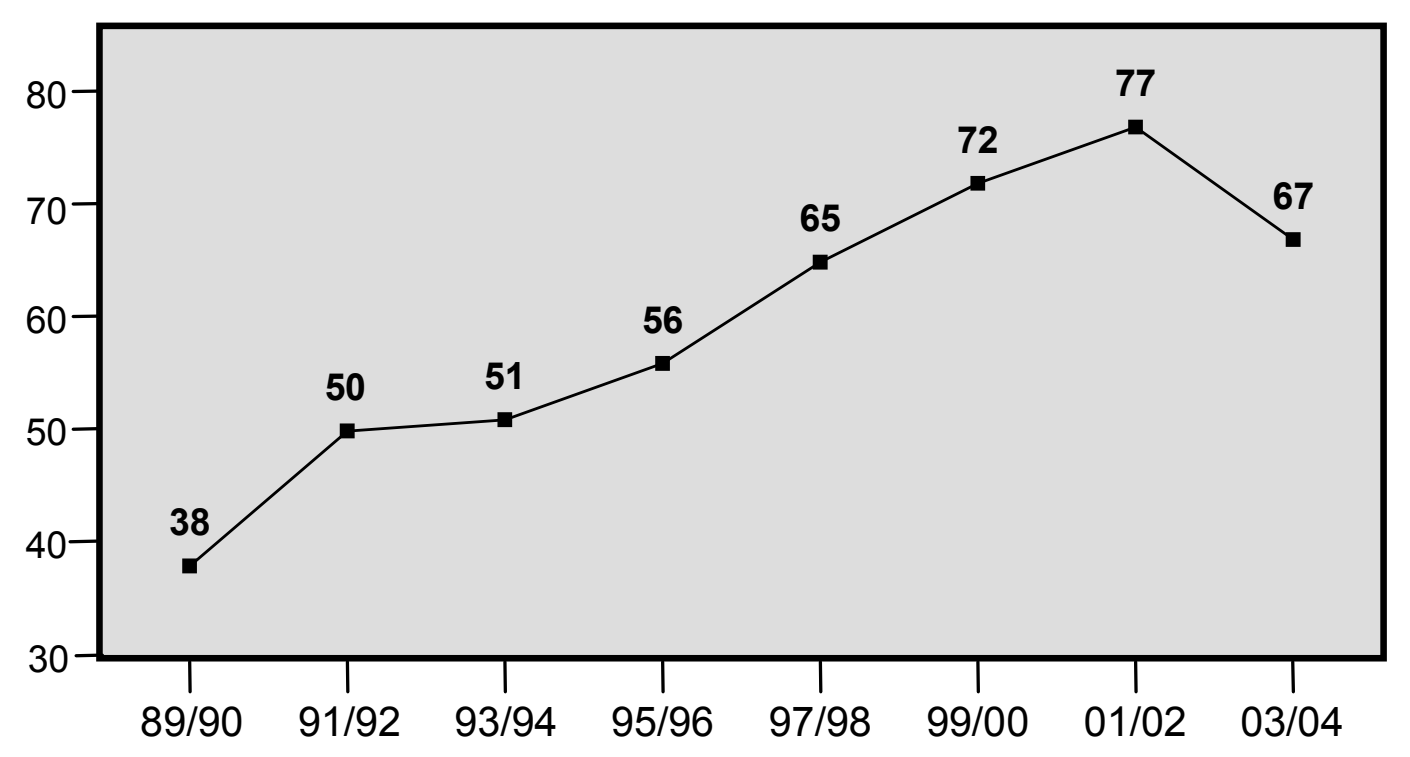

ausgewertet wurden folgende 12 Zeitschriften:

BFuP, CM, Controlling, DB, DBW, io new management, WiSt, WISU, ZfB, zfbf, ZfCM, zfo

Abb. 1: Anzahl NPO-bezogener Artikel in ausgewählten betriebswirtschaftlichen Zeitschriften von 1989-2004

Quelle: Eigene Darstellung

Ein Schwerpunkt der Veröffentlichungen zu NPOs liegt auf Controlling-spezifischen Aspekten. Diesbezüglich werden große Verbesserungspotenziale in der Praxis vermutet. Das Controlling für NPOs steht allerdings nicht allein deshalb im Mittelpunkt der folgenden Überlegungen; es wird hier auch thematisiert, weil es als Forschungsfeld der Betriebswirtschaftslehre noch eklatante, v.a. methodische Lücken aufweist.

Dies soll exemplarisch am Beispiel des Krankenhaussektors, insbesondere des dortigen Einsatzes der Balanced Scorecard, verdeutlicht werden. Ziel ist es einerseits, die Praktiker für die Thematik zu sensibilisieren. Andererseits soll dazu angeregt werden, nicht unbedacht den „Versuch eines Know-How-Transfers von etablierten Erkenntnissen aus dem Einsatz privatwirtschaftlich orientierter Controlling-Instrumente ${ }^{\text {“1 } 1} \mathrm{zu}$ unternehmen, sondern die Forschung auf dem Gebiet des NPO-Controllings voranzutreiben.

\section{Zum Anpassungsbedarf des Controlling-Instrumentariums}

NPOs grenzen sich von rein privatwirtschaftlich geführten Unternehmen durch eine Reihe von Aspekten ab, v.a. durch andere Rahmenbedingungen, Strukturen und Zielsetzungen. ${ }^{2}$ Folglich ist der Einsatz traditioneller Controlling-Instrumente, in denen solche Aspekte eine maßgebliche Rolle spielen, systematisch zu hinterfragen. Die Ergebnisse entsprechender Analysen können unterschiedlicher Natur sein. Es kann sich herausstellen,

$1 \quad$ Klingebiel (1999), S. 378.

2 Vgl. im Einzelnen Bermann (2004), S. 230 f. 
dass ein Instrument unverändert einsetzbar ist, dass es nur wenig bzw. grundlegend angepasst werden muss oder dass es sogar untauglich ist. Diesen kritischen Umgang mit den Instrumenten lässt allerdings so mancher Controlling-bezogener Literaturbeitrag zu NPOs vermissen.

Hier seien exemplarisch (nicht privat geführte) Krankenhäuser betrachtet, nicht zuletzt weil sie in allen drei oben genannten Aspekten - Rahmenbedingungen, Strukturen und Zielsetzungen - Besonderheiten gegenüber privatwirtschaftlich geführten Unternehmen aufweisen. So ist das Management von Krankenhäusern aufgrund des öffentlichen Versorgungsauftrags dadurch eingeschränkt, dass die angebotenen Leistungen nicht beliebig gestaltbar sind. Schwierigkeiten bereitet zudem die starke organisatorische, inhaltliche wie auch gelebte Trennung zwischen kaufmännischem und medizinischem Bereich. Eine weitere Herausforderung stellt schließlich die mit dem Versorgungsauftrag einhergehende breitere Fächerung von Sachzielen (lückenlose medizinische Versorgung, patientengerechte Betreuung etc.) und ihre höhere Bedeutung gegenüber Formalzielen dar.

In diesem Kontext ist zu hinterfragen, inwieweit klassische (Controlling-)Instrumente auch im Krankenhaus einsetzbar sind. Beispielsweise finden sich in der Literatur Vorschläge zur Anwendung der Portfolio-Analyse, ${ }^{3}$ ohne dass dabei das Grundkonzept kritisch reflektiert würde. Diesbezüglich drängen sich unter anderem folgende Fragen auf: ${ }^{4}$

- Inwieweit lassen sich Sachziele wie die oben angeführten in die üblicherweise auf den Portfolio-Achsen abgetragenen Kriterien integrieren?

- Lassen sich genügend Geschäftsfelder identifizieren, für welche die der PortfolioAnalyse zugrunde liegenden Konzepte des Produktlebenszyklus und der Erfahrungskurve Gültigkeit haben?

- Ist auf dieser Basis die Portfolio-Idee einer Innenfinanzierung im Rahmen des finanziellen Ausgleichs zwischen unabhängigen strategischen Geschäftsfelder umsetzbar?

Auf diese Fragen soll hier keine Antwort gegeben werden. Ziel ist es vielmehr, die Problematik einer unkritischen Übertragung klassischer Controlling-Instrumente auf den NonProfit-Sektor beispielhaft zu verdeutlichen. Diesem Ziel dienen auch die folgenden, mit neuen empirischen Erkenntnissen untermauerten Ausführungen zur Balanced Scorecard (BSC). Mit Blick auf die zahlreichen Veröffentlichungen zur BSC wird dabei auf eine detaillierte Erläuterung ihrer vierstufigen Methodik (1. Strategiekonkretisierung, 2. Strategiekommunikation, 3. Budgetierung und Kontrolle, 4. Strategisches Lernen) verzichtet. $^{5}$

3 Vgl. Greiling/Jücker (2003), S. 120 f.; Schirmer (2003), S. 125 ff.

4 Vgl. auch die Kritik von Braun von Reinersdorff (2002), S. 259 ff.

5 Zu einer umfassenden Beschreibung der BSC vgl. Kaplan/Norton (1997); im Hinblick auf eine kritische Erörterung der BSC und weiterführende Literatur sei auf Ahn (2003) verwiesen. 


\section{Die Balanced Scorecard im Krankenhaus}

Im Frühjahr 2005 wurde eine Online-Befragung aller deutschen Krankenhäuser mit mindestens 250 Betten zum Stand des Einsatzes der BSC durchgeführt. Neben grundlegenden Ergebnissen ${ }^{6}$ lässt sich aus der Studie schließen, dass das originäre BSC-Konzept in der Krankenhauspraxis nicht trägt. So wird das BSC-Konzept meist nur rudimentär umgesetzt. Beispielsweise besitzen von den 70 Krankenhäusern, deren Antworten verwertbar waren, lediglich 20\% Regelungen zur Strategiekommunikation (Stufe 2 der BSCMethodik) und lediglich 15,7\% Regelungen zum Strategischen Lernen (Stufe 4 der BSCMethodik). Man ist geneigt zu mutmaßen, dass dies mit der Besonderheit der strikten Trennung zwischen kaufmännischem und medizinischem Bereich zu tun hat. ${ }^{7}$

Unabhängig davon lässt sich die mangelnde Tragfähigkeit des originären BSC-Konzepts für den Krankenhaussektor an einem weiteren Aspekt festmachen, welcher den Kern der BSC-Methodik betrifft. Dieser Kern manifestiert sich zum einen in der Idee, zwecks Strategiekonkretisierung die zentralen Zielsetzungen einer Organisation(seinheit) aus vier vorformulierten Perspektiven heraus abzuleiten; die in Abbildung 2 wiedergegebenen Perspektiven bilden eine Hierarchie, auf deren oberster Ebene allein die Finanzperspektive angesiedelt ist. Zum anderen sind die den Perspektiven zuzuordnenden strategischen Ziele über plausible Ursache-Wirkungs-Vermutungen miteinander zu verknüpfen. Dabei sind von der Potenzial- bis hin zur Finanzperspektive durchgängige Ursache-WirkungsKetten zu bilden (vgl. das Beispiel in Abbildung 2). Darin kommt zum Ausdruck, dass letztendlich alle Ziele der unteren drei Ebenen der Verbesserung der Ziele auf der Finanzperspektive dienen sollen.

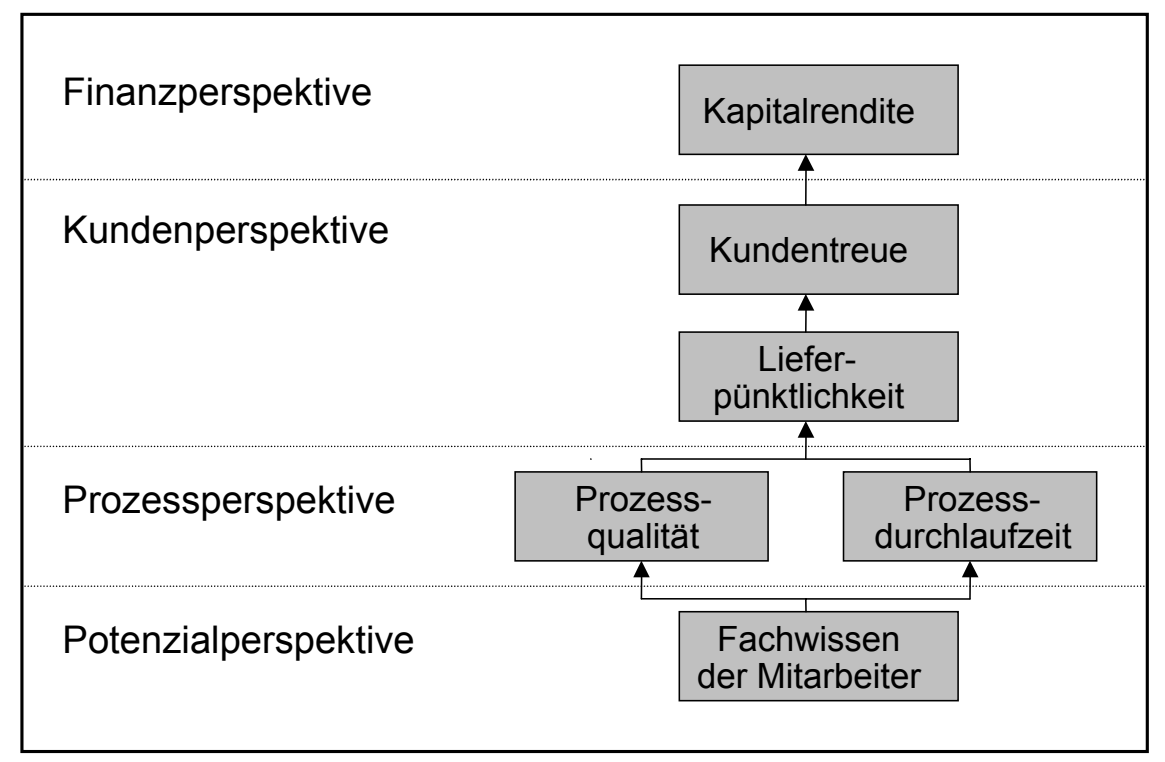

Abb. 2: Das Ursache-Wirkungs-Konzept der BSC

Quelle: Kaplan/Norton (1997), S. 29

6 Vgl. dazu Ahn/Reuter (2005).

7 Allerdings gibt es auch empirische Hinweise darauf, dass die BSC ebenso in privatwirtschaftlich geführten Unternehmen eine geringe Umsetzungstiefe aufweist; vgl. dazu Speckbacher/Bischof/Pfeiffer (2003), S. 372 f. 
Für Krankenhäuser weist dieses zentrale BSC-Element hierarchisch angeordneter, über Ursache-Wirkungs-Vermutungen verbundener Perspektiven und Ziele mit allein der Finanzperspektive auf oberster Ebene gleich mehrere - für NPOs typische - Fallstricke auf:

1. Die breitere Fächerung von Sachzielen legt eine inhaltliche Umdeutung der vorformulierten Perspektiven bzw. die Definition neuer Perspektiven nahe.

2. Die wesentliche höhere Bedeutung von Sachzielen stellt die alleinige Positionierung der Finanzperspektive auf oberster Ebene in Frage.

3. Damit einhergehend wird die Verknüpfung der den Perspektiven zugeordneten Ziele über durchgängige Ursache-Wirkungs-Ketten bei (wahrscheinlichen) Zielkonflikten zwischen den auf oberster Ebene angesiedelten Perspektiven erschwert. Die nahe liegende Konsequenz daraus ist, auf eine hierarchische Anordnung der Perspektiven ganz zu verzichten.

Diese drei Aussagen haben zunächst einmal nur den Charakter von Thesen, lassen sich aber empirisch untermauern. So sprechen zahlreiche Einzelbeispiele aus der Krankenhauspraxis ${ }^{8}$ für die These 1. Zudem gaben in der durchgeführten Umfrage immerhin 16 von 50 Krankenhäuser an, in ihrer BSC die Perspektivenanzahl von 4 auf bis zu 9 Perspektiven erhöht zu haben. ${ }^{9}$

These 2 findet ihre Bestätigung in Abbildung 3. Nur gut jedes zehnte Krankenhaus belässt es beim klassischen Ansatz, die Finanzperspektive allein auf oberster Ebene zu positionieren. Dagegen wird dieser Ansatz in der weit überwiegenden Zahl der Fälle $(71,2 \%)$ dahingehend verändert, dass mindestens eine weitere Perspektive auf oberster Ebene angesiedelt wird. Abbildung 3 gibt auch bereits einen Hinweis in Bezug auf These 3 . Schließlich stellen immerhin 12,1\% der untersuchten Krankenhäuser die Idee hierarchisch aufeinander aufbauender Perspektiven zur Disposition, indem sie die Finanzperspektive allen Ebenen gleichzeitig zuordnen.

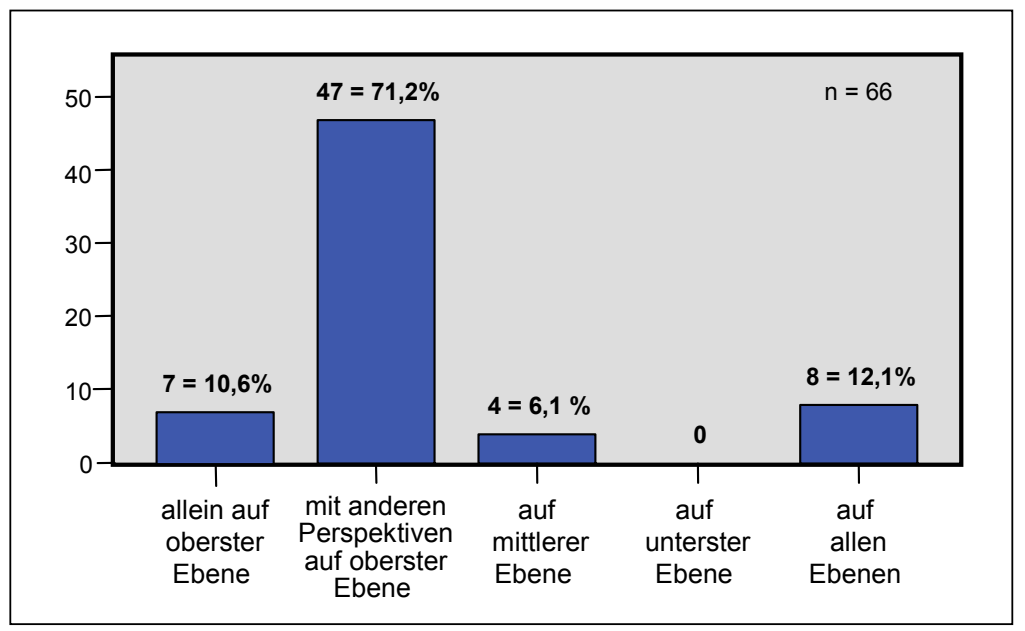

Abb. 3: Anordnung der Finanzperspektive in BSCs von Krankenhäusern

Quelle: Eigene Darstellung

8 Vgl. stellvertretend z.B. Greulich u.a. (2002), S. 155 ff.; Roth (2002), S. 250 ff.

9 Nicht möglich - weil nicht abgefragt - ist eine Aussage darüber, inwieweit inhaltliche Umdeutungen der vorformulierten Perspektiven erfolgt sind. 
Dass These 3 zutreffend ist, untermauert Abbildung 4. In ihr sind die Antworten auf die Fragen nach der Anzahl der BSC-Perspektiven einerseits und der Anzahl der Perspektiven auf oberster Ebene andererseits gegenübergestellt. Zwar haben nur 29 Krankenhäuser beide Fragen zugleich auswertbar beantwortet, das Ergebnis ist aber doch recht eindeutig. So zeigt die Diagonale in Abbildung 4 an, wie viele Krankenhäuser sämtliche ihrer Perspektiven auf oberster Ebene anordnen - und sich damit gänzlich von der hierarchischen Verknüpfung dieser Perspektiven verabschieden. Relativ ausgedrückt geschieht dies in nicht weniger als 79,3\% der Fälle.

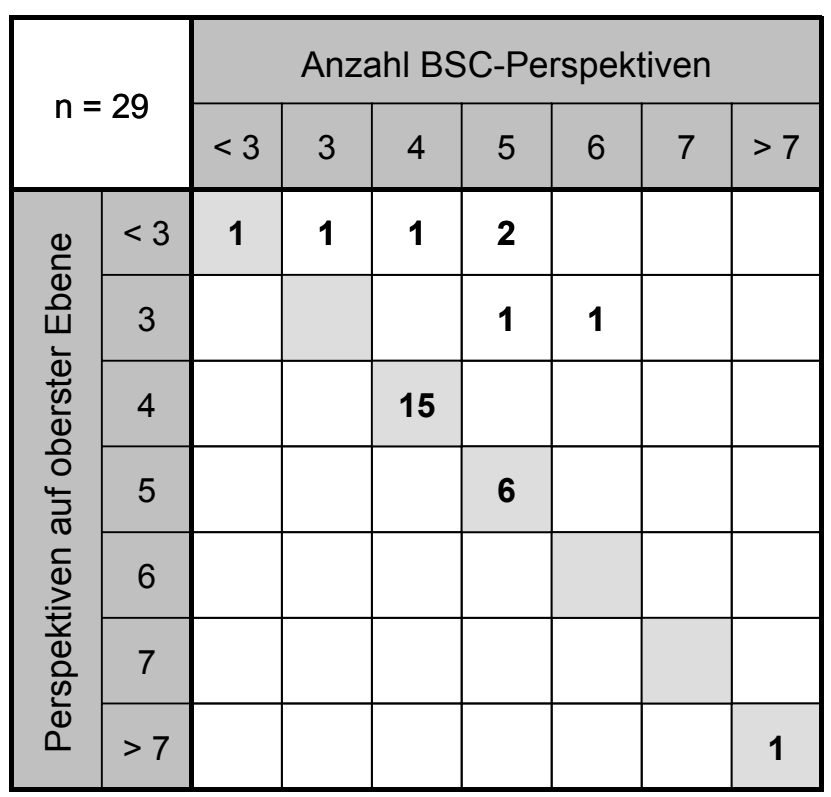

Abb. 4: Verteilung der Krankenhäuser hinsichtlich ihrer Angaben zu „Anzahl BSCPerspektiven “ und „Perspektiven auf oberster Ebene“

Quelle: Eigene Darstellung

\section{Konsequenzen}

Die empirischen Ergebnisse lassen befürchten, dass im Krankenhaussektor - und vermutlich auch in anderen Non-Profit-Bereichen - in vielen Fällen Ansätze verfolgt werden, die nur noch entfernt an das ursprüngliche BSC-Konzept erinnern. Dies ist insofern zu bedauern, als dabei wesentliche Stärken der BSC gegenüber herkömmlichen Ziel- und Kennzahlensystemen nicht mehr zum Tragen kommen können. Um dem entgegenzusteuern, sind NPO-spezifische Weiterentwicklungen des BSC-Konzepts notwendig, zum Beispiel hinsichtlich der methodischen Unterstützung bei

- der Ableitung der im jeweiligen Einzelfall relevanten Perspektiven,

- der Strukturierung dieser Perspektiven, insbesondere in Bezug auf die Positionierung der Finanzperspektive, und

- $\quad$ der Bewältigung von Konflikten zwischen prinzipiell gleichberechtigten Zielen, die spätestens im Rahmen der Prioritätensetzung hinsichtlich der zu ergreifenden Maßnahmen auftreten. 
Über diese Beispiele hinaus will dieser Beitrag nicht nur grundsätzlich dazu anregen, auf die Erfordernisse von NPOs ausgerichtete Controlling-Instrumente zu entwickeln. Die Ausführungen sollen auch als Denkanstoß verstanden werden, wie das Forschungsfeld NPO insgesamt belebt werden kann. Diesbezüglich sind die Praktiker insofern in die Pflicht zu nehmen, als sie propagierten Managementtechniken kritisch(er) gegenübertreten. Ihnen sollte bewusst sein, dass ,sich die Lösungsansätze der 'klassischen' Betriebswirtschaftslehre aufgrund der Besonderheiten des Krankenhaussektors [im Speziellen bzw. des NPO-Sektors im Allgemeinen] nicht friktionslos auf diesen übertragen lassen“. ${ }^{10}$

Controlling für NPOs stellt insoweit ein relevantes Forschungsfeld dar, als man sich auf dem Weg zu eigenständigen Konzepten, Ansätzen oder gar Theorien befindet. Hier wird dazu aufgerufen, diesen Weg konsequenter zu beschreiten. Anderenfalls droht ein Bedeutungsverlust des Forschungsfelds. Ein erstes Indiz dafür lässt sich der in Abbildung 1 wiedergegebenen zeitlichen Entwicklung der Häufigkeit von NPO-Veröffentlichungen entnehmen. Zusammen mit dem sich für 2005 abzeichnenden weiteren Rückgang der Publikationshäufigkeit erinnert die dargestellte Kurve in ihrem Verlauf stark an einen idealtypischen Produktlebenszyklus. Diesbezüglich scheint die Phase einer ersten Sättigung erreicht zu sein. Es kommt nun darauf an, einen erfolgreichen Relaunch in die Wege zu leiten.

\section{Literaturverzeichnis}

Ahn, Heinz (2003), Effektivitäts- und Effizienzsicherung - Controlling-Konzept und Balanced Scorecard, Frankfurt am Main u.a.

Ahn, Heinz und Stefan Reuter (2005), Balanced Scorecard fasst in Krankenhäusern nur langsam Fuß, in: f\&w führen und wirtschaften im Krankenhaus, 22. Jg., Nr. 6, S. 614-616

Bergmann, Michael (2004), Balanced Scorecard in Non-Profit-Organisationen - Einsatzmöglichkeiten und Adaptionserfordernisse, in: Controlling, 16. Jg., Nr. 4/5, S. 229-236

Braun von Reinersdorff, Andrea (2002), Strategische Krankenhausführung - Vom Lean Management zum Balanced Hospital Management, Bern u.a.

Greiling, Michael und Carolin Jücker (2003), Strategisches Management im Krankenhaus - Methoden und Techniken zur Umsetzung in der Praxis, Stuttgart

Greulich, Andreas u.a. (2002), Balanced Scorecard im Krankenhaus - Von der Planung bis zur Umsetzung, Heidelberg

Helmig, Bernd und Dieter K. Tscheulin (1998), Krankenhausmanagement in der deutschsprachigen betriebswirtschaftlichen Forschung im internationalen Vergleich - Eine Bestandsaufnahme, in: Zeitschrift für Betriebswirtschaft, 68. Jg., Nr. 1, S. 83-110

Kaplan, Robert S. und David P. Norton (1997), Balanced Scorecard - Strategien erfolgreich umsetzen, Stuttgart

Klingebiel, Norbert (1999), Steuerungserfordernisse in Non-Profit Organisationen, in: Kostenrechnungspraxis, 43. Jg., Nr. 6, S. 372-379

Roth, Sonja (2002), Entwicklung einer Balanced Scorecard als strategisches Steuerungsinstrument in einem öffentlichen Klinikum, in: Balanced Scorecard in Verwaltung und Non-Profit-Organisationen, hrsg. von Andreas Georg Scherer und Jens Michael Alt, Stuttgart, S. 239-263

Schirmer, Herbert (2003), Krankenhaus-Controlling - Handlungsempfehlungen für Krankenhausmanager und Krankenhauscontroller, 2. Aufl., Renningen

Speckbacher, Gerhard, Jürgen Bischof und Thomas Pfeiffer (2003), A Descriptive Analysis on the Implementation of Balanced Scorecards in German-Speaking Countries, in: Management Accounting Research, 14. Jg., Nr. 4, S. 361-387

10 Helmig/Tscheulin (1998), S. 95. 